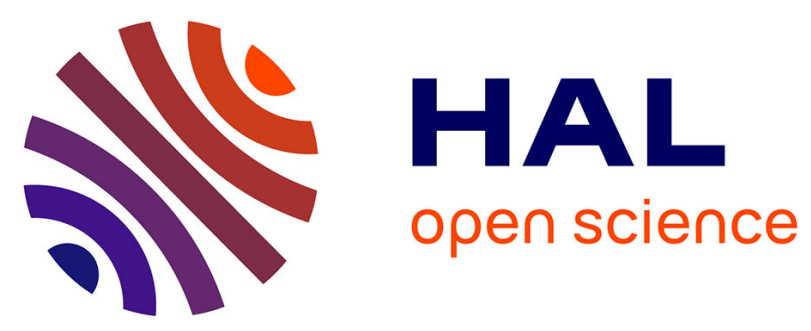

\title{
Implementation of atlas-matching capabilities using web services technology: lessons learned from the development of a demonstrator
}

\author{
Bernard Gibaud, Olivier Dameron, Eric Poiseau, Pierre Jannin
}

\section{To cite this version:}

Bernard Gibaud, Olivier Dameron, Eric Poiseau, Pierre Jannin. Implementation of atlas-matching capabilities using web services technology: lessons learned from the development of a demonstrator: Implementation of atlas-matching capabilities using web services technology: lessons learned from the development of a demonstrator. Jan 2005, France. pp.266-271. inserm-00137672

HAL Id: inserm-00137672 https://www.hal.inserm.fr/inserm-00137672

Submitted on 11 May 2010

HAL is a multi-disciplinary open access archive for the deposit and dissemination of scientific research documents, whether they are published or not. The documents may come from teaching and research institutions in France or abroad, or from public or private research centers.
L'archive ouverte pluridisciplinaire HAL, est destinée au dépôt et à la diffusion de documents scientifiques de niveau recherche, publiés ou non, émanant des établissements d'enseignement et de recherche français ou étrangers, des laboratoires publics ou privés. 


\title{
Implementation of atlas-matching capabilities using "web services" technology: lessons learned from the development of a demonstrator
}

\author{
Bernard Gibaud $^{\mathrm{a}}$, Olivier Dameron ${ }^{\mathrm{a}}$, Eric Poiseau ${ }^{\mathrm{a}}$, Pierre Toulouse ${ }^{\mathrm{a}}$ \\ and Pierre Jannin \\ ${ }^{a}$ Labo IDM, UPRES 3192 Inserm ESPRI, Faculté de médecine, Rennes, France \\ bernard.gibaud@univ-rennes1.fr
}

\begin{abstract}
In this paper, we propose an approach for sharing data processing functions such as image to atlas registration and matching, based on platform-independent interfaces called "web services". A prototype implementing this concept is described, enabling a user to match image data, e.g. MRI, to the Talairach atlas, as well as to 3D-data atlases such as the Statistical Probability Anatomy Maps (SPAM) datasets developed by the Montréal Neurological Institute (MNI). We emphasize architectural issues rather than functional aspects, which we consider classical.
\end{abstract}

Keywords: brain atlas, web technology, web services

\section{Introduction}

In the context of neuroimaging, it is often useful to match anatomical (e.g. MRI, CT) or functional (e.g. fMRI, PET, MEG/EEG) observations with a priori knowledge about brain anatomy and function. Representing this knowledge and implementing tools achieving this matching [1] usually relies on specific and ad hoc approaches, leading to a significant duplication of effort. Today, the progress of communication infrastructures and techniques, and particularly the Internet, enables a widespread sharing and reuse of data and knowledge, by both humans and machines, through platform-independent interfaces (e.g. web services). This kind of technology is more and more used in commerce and industry, as a result of the development and promotion activities of large organizations such as the Organization for the Advancement of Structured Information Standards (OASIS). It is clear that the medical image computing field could certainly benefit of components and standards developed in these contexts.

We hypothesized that matching anatomical or functional observations with a priori knowledge about the brain involves some common functions, and that these functions are relevant in several applications (e.g. clinical practice, research, education). Typically, such functions involve aligning a subject's set of images with a reference coordinate system (e.g. the Talairach reference coordinate system), and retrieving atlas plates corresponding to a particular point selected in one of the subject's images. 
We revisited the classical problem of atlas matching and propose an architecture based on web services for providing unified access to two kinds of brain atlases, namely:

(1) plates-based atlases such as the Talairach atlas for brain anatomy [2], and an atlas of Brodmann areas derived from Damasio's work [3] and available in the distribution of the MRIcro software (Chris Rorden) [4],

(2) 3D-data atlases such as the Statistical Probability Anatomy Maps (SPAM) datasets developed by the Montréal Neurological Institute (MNI). The SPAM provide statistical information about the position (in a standard space) of 98 brain structures such as cortical gyri, basal ganglia, lobes, etc. Each anatomical structure is represented by a $3 \mathrm{D}$ dataset, in which each voxel denotes the probability of presence of this structure at this particular voxel (for details about the SPAM building process, see [5]). Thresholding of this volume (e.g. at a 0.5 or 0.75 probability value) allows a 3D surface to be defined, that can then be displayed, thus providing the user with an estimate of the position and shape of this structure in space.

The primary goal of this prototyping work was to assess the relevance of this approach (complexity, actual reuse-ability of the web services, etc.)

\section{Methods}

\subsection{Architecture}

The proposed architecture involves client applications that invoke remote services implemented as web services, i.e. that are accessible through internet thanks to the Simple Object Access Protocol (SOAP) over HTTP. The primary advantage of this approach is that many client applications can share the processing tools implemented as web services.

\subsection{Functional aspect}

Two major services were defined :

- $\quad$ retrieval of the three atlas plates (in the sagittal, coronal and axial planes) corresponding to a 3D point of interest specified by the client application, together with the name of the atlas to be used. The coordinates of this 3D point may be represented either in a "client coordinate system", or in a "standard coordinate system";

- Retrieval of the SPAM surfaces, in response to a request from the client application specifying the anatomical structure to be retrieved (SPAM name) and the level of thresholding to be used (probability $>0.0 ; 0.5 ; 0.75$ ).

In our work, one of the common services consisted in calculating and applying geometrical transformations between a "client coordinate system" and a "standard coordinate system". The "client coordinate system" was defined by the client application; in our prototype, it was usually defined by the MRI dataset that the user wanted to match with the atlases. The "standard coordinate systems" was "the Talairach and Tournoux reference coordinate system” (as defined in the 1988 atlas [2]) and the 
"MNI coordinate system" [6]: the former was defined by the Talairach coordinate system fiducials (namely, a list of three or more points belonging to the interhemispheric plane, the anterior commissure, the posterior commissure, and the right and left hemispheres' most anterior, most posterior, most superior and most inferior points); the latter was defined with respect to the former by an affine transformation [6].

\subsection{Client applications}

We considered two important application contexts:

(1) the direct consultation of atlas plates by a user using a web browser,

(2) the consultation of atlas plates and SPAM data in a complex application, dedicated to the segmentation and display of MRI data. This application was initially developed to segment cortical sulci, as part of the preparation of neurosurgical procedures involving multimodal neuronavigation.

Since the same atlas matching capabilities were involved, an important feature was then to be able to reuse the same components in both cases.

\section{Results}

A set of web services was designed and implemented (in PHP). The most important ones were:

- function sessionCreation() : to create a session context on the server side

- function logout() : to release the session context on the server side

- function build_client_referential() : to store in the session context the characteristics of the client coordinate system (based on the Talairach coordinate system fiducials provided by the client application)

- function get_result_atlas() : to retrieve the atlas plates and the related cursor positions, corresponding to a particular 3D point

- function get_spam_surface() : to retrieve the surface of a particular SPAM

The implementation of the web services was made using the NuSOAP (dietrich.ganx4.com/nusoap/index.php) and PySOAP (use of SOAP in programs written in Python) libraries.

Implementation of the direct consultation of atlas plates through a web browser made it necessary to develop a suitable presentation server (written in PHP), accessible in HTTP. It was responsible for requesting the atlas plates from the web services server using SOAP messages, and to put the information together as html pages that were then served to the web browser by HTTP (Fig. 1). 


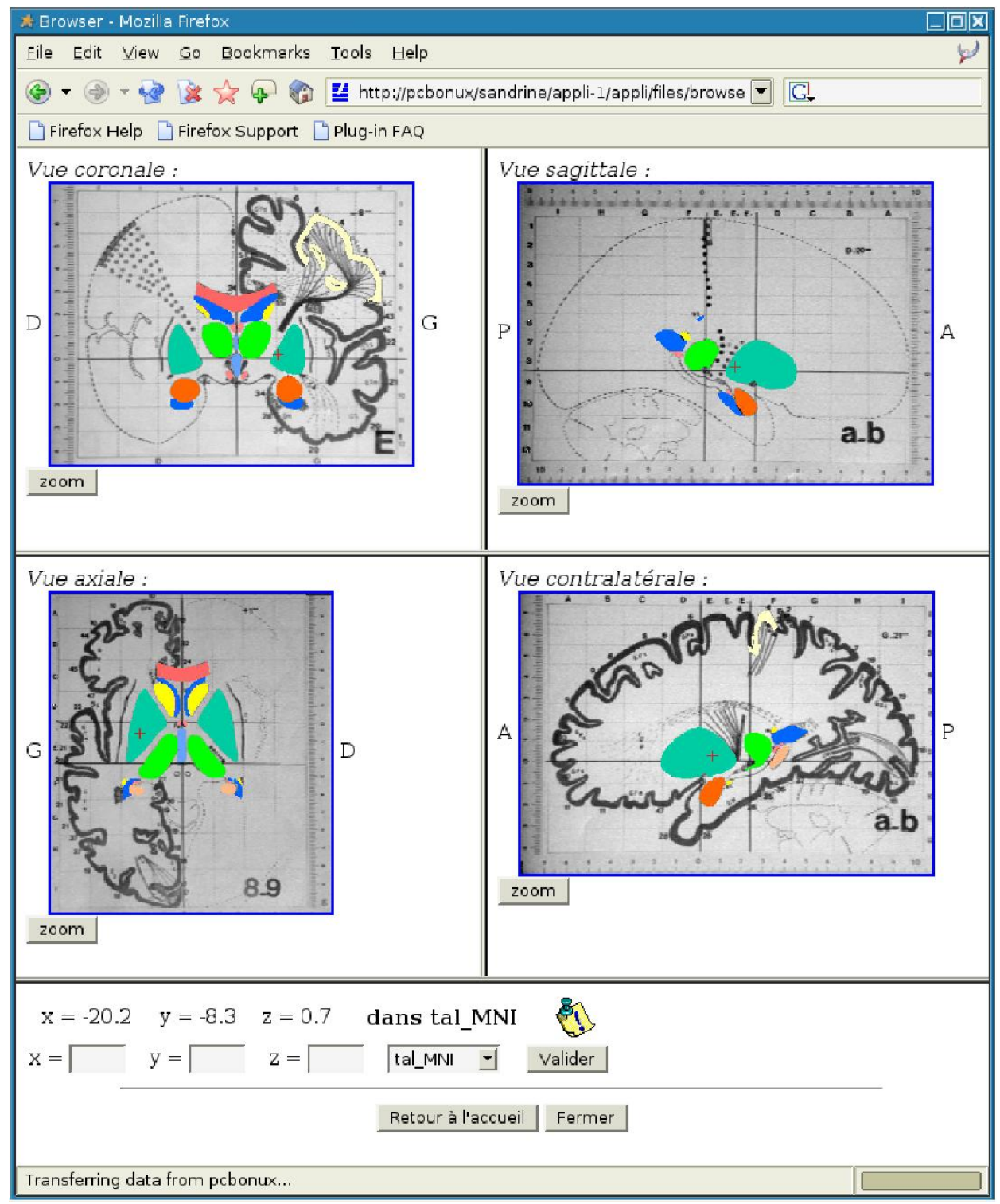

Fig. 1. HTML page generated by the presentation server, for consultation of the Talairach atlas using a web browser: coronal view (top left), axial view (bottom left), sagittal view (top right) and contralateral sagittal view (bottom right).

In the second application (written in Python), all presentation and user interaction aspects were directly managed by the application (Fig. 2). 


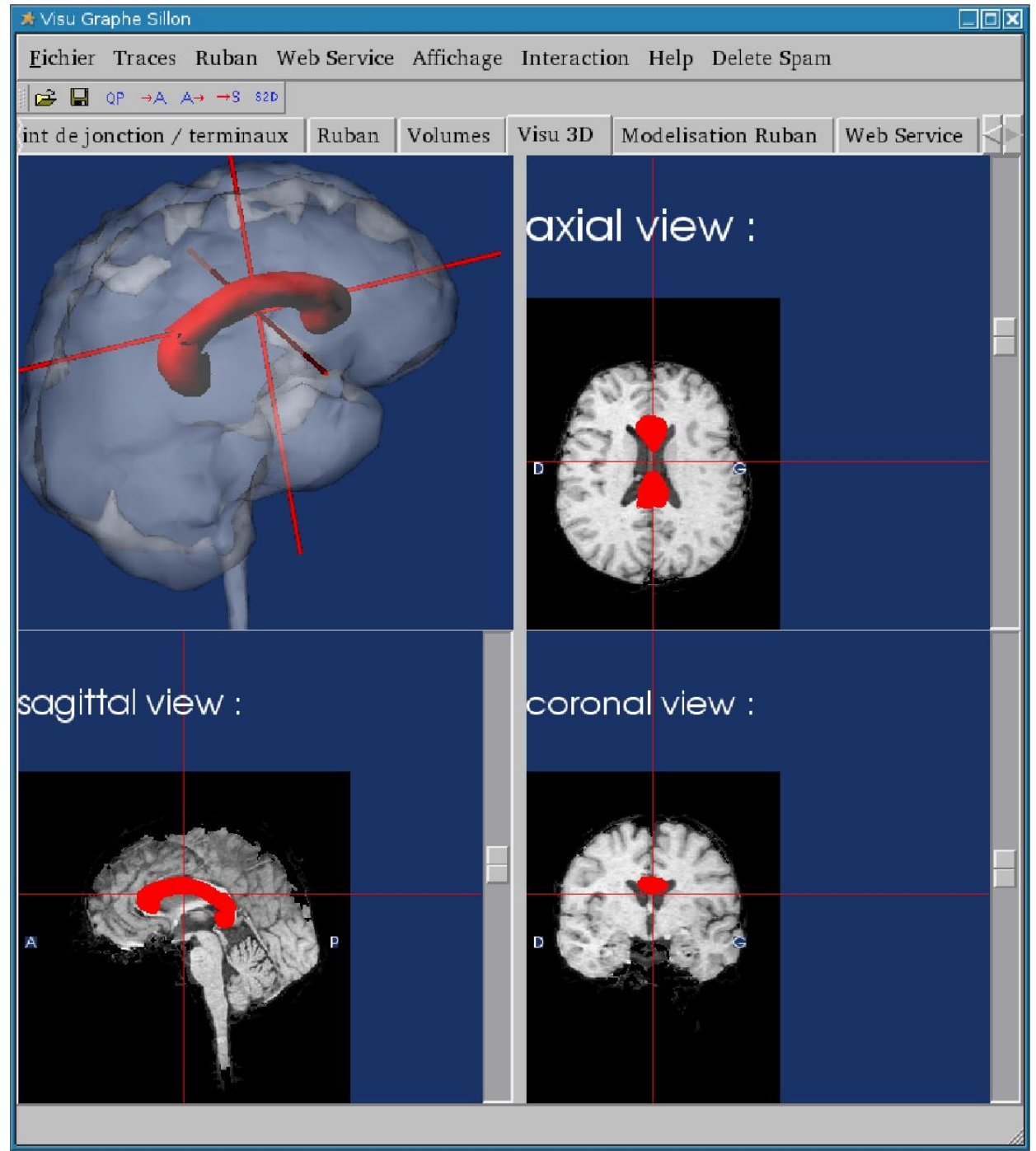

Fig. 2. Segmentation and display of MRI images: the top left image shows a SPAM structure retrieved using the web services (corpus callosum in red).

\section{Conclusion}

The prototypes were deployed in our research lab’s intranet. At this stage, their assessment was made from a pure technical point of view. It means that any discussions about the functional added value, or regarding precision of registration were out of our scope. Performance was found satisfactory in the sense that atlas plates (represented in JPEG and encoded in Base64) were transferred and displayed without significant delay. 
The implementations of SOAP that we have used were both fast and reliable. Security issues have not been addressed so far. It is clear that deployment of our web services over the internet will make it necessary to implement authentication and access control mechanisms.

This feasibility study allowed to demonstrate the ability of web services to integrate components developed in very different environments (e.g. Python on the client side, and PHP on the server side), as well as the reuse-ability of the web services.

Other lessons learned from this prototype implementation concerned the definition of the interface, especially regarding the services' granularity: actually, a tradeoff had to be found between numerous atomic "ancillary services", with high reuse potentialities, and more specific "application-level services", probably easier to understand and use.

We believe that such approaches, based on the availability of image processing services, may change significantly the way applications are developed and integrated in the medical imaging field. From a research perspective, it raises new questions concerning the interoperability of components, e.g. about the way to represent interoperability contraints. In this regard, the creation of a new working group in the DICOM arena (DICOM working group 23 "application hosting”) is highly significant.

\section{Acknowledgements}

The authors gratefully acknowledge Louis Collins and Alan Evans for providing the SPAM data, Brian Scanlan (Thieme) for giving permission to use the Talairach's plates contained in the Talairach and Tournoux atlas in our demonstrator, and Sandrine Di Meo and Damien Le Blé for their contributions in the implementation.

\section{References}

[1] Gibaud B, Garlatti S, Barillot C, Faure E. Computerized brain atlases as decision support systems: a methodological approach, Artificial Intelligence in Medicine 1998;14:83-100.

[2] Talairach J, Tournoux P. Co-planar stereotactic atlas of the human brain, Thieme, 1988.

[3] Damasio H and Damasio AR., Lesion Analysis in Neuropsychology, New Yourk, Oxford, 1989.

[4] Rorden C, MRIcro, http://www.psychology.nottingham.ac.uk/staff/cr1/template.html

[5] Collins DL, Zijdenbos AP, Evans AC. Improved automatic gross cerebral structure segmentation, 4th International Conference on Functional Mapping of the Human Brain, Montréal, 1998.

[6] http://www.mrc-cbu.cam.ac.uk/Imaging/mnispace.html 